\title{
EFEITOS DA REEDUCAÇÃO POSTURAL GLOBAL (RPG) SOBRE A HIPERCIFOSE TORÁCICA: UM ESTUDO DE CASO
}

\author{
Luana Machado Moreira ${ }^{1}$ \\ Juliana Adami Sedrez ${ }^{2}$ \\ Matias Noll ${ }^{3}$ \\ Claudia Tarragô Candotti ${ }^{4}$
}

MOREIRA, L. M.; SEDREZ, J. A.; NOLL, M.; CANDOTTI, C. T. Efeitos da reeducação postural global (rpg) sobre a hipercifose torácica: um estudo de caso. Arq. Cienc. Saúde UNIPAR, Umuarama, v. 21, n. 2, p, 113-117, maio/ago. 2017.

\begin{abstract}
RESUMO: A Reeducação Postural Global (RPG) vem sendo empregada na prevenção e reabilitação de alterações posturais, principalmente desordens da coluna vertebral, mas seus efeitos no tratamento da hipercifose torácica ainda são pouco descritos na literatura. O objetivo do estudo foi verificar os efeitos do tratamento de RPG em um sujeito do sexo feminino com hipercifose torácica. O paciente foi submetido a 10 sessões de uma hora do método RPG. As sessões foram compostas por duas posturas: postura "rã no chão de braços abertos" e postura "em pé contra a parede". Os dados foram coletados com o instrumento flexicurva antes do início do tratamento (pré-teste), antes e após cada sessão de tratamento, e uma semana após a última sessão do tratamento (pós-teste), sendo obtidos os ângulos das curvaturas da coluna vertebral. Foi realizado análise descritiva com média, desvio-padrão e frequências. Para verificar se houveram diferenças entre as avaliações antes e após cada sessão, foi realizada a média das avaliações e comparadas a partir do teste de Mann-Whitney. As análises foram realizadas no software SPSS $17.0(\alpha=0,05)$. Os resultados do presente estudo demonstraram que o tratamento de RPG foi eficaz, em média, após cada sessão de tratamento, na diminuição imediata da hipercifose torácica $(U=23,5 ; p=0,045, r=0,45)$. Verificou-se também que o tratamento de RPG de 10 semanas foi eficaz na diminuição da hipercifose torácica, ou seja, redução do ângulo da cifose torácica de $53,9^{\circ}$ para $49^{\circ}$. Conclui-se que a RPG apresentou efeito agudo de redução da hipercifose torácica no caso estudado, após cada sessão de tratamento e pós 10 semanas de intervenção.
\end{abstract}

PALAVRAS-CHAVE: Coluna vertebral. Fisioterapia. Postura.

\section{EFFECTS OF GLOBAL POSTURE RE-EDUCATION (GPR) ON THORACIC HYPERKYPHOSIS: A CASE STUDY}

\begin{abstract}
Global Posture Re-education (GPR) has been used in the prevention and rehabilitation of postural alterations, especially in disorders of the spine, although its effects in the treatment of thoracic hyperkyphosis are still poorly described in the literature. The aim of this study was to verify the effects of GPR treatment in a female subject with thoracic kyphosis. The patient underwent 10 one-hour sessions of GPR. The sessions were composed of two postures: the "frog on the floor with open arms" posture and "standing against the wall" posture. Data were collected using the flexicurve instrument before the beginning of the treatment (pre-test), before and after each treatment and one week after the last treatment session (post-test), obtaining measurements from the curvature angles of the spine. A descriptive analysis was performed with mean, standard deviation and frequencies. In order to check whether there were differences between measurements before and after each session, the session average was calculated and compared from the Mann-Whitney test. The analyses were performed using the SPSS 17.0 software $(\alpha=0.05)$. The results of the study showed that the GPR treatment was effective, on average, after each treatment session, in the immediate decrease in thoracic kyphosis $(U=23,5 ; p=0,045, r=0,45)$. It was also found that a 10 -week GPR treatment was effective in reducing thoracic kyphosis, that is, it reduced the thoracic kyphosis angle from $53.9^{\circ}$ to $49^{\circ}$. In conclusion, GPR presented an acute effect on the reduction of thoracic hyperkhphosis in the studied case after each treatment session and after 10 weeks of intervention.
\end{abstract}

KEYWORDS: Physical therapy. Posture. Spine.

\section{Introdução}

A técnica de Reeducação Postural Global (RPG) parte do pressuposto de que um músculo encurtado cria compensações em músculos proximais ou distais e fundamenta-se em gerar um alongamento ativo simultâneo dos músculos pertencentes à mesma cadeia muscular (LAWAND et al., 2013). Para isso, o criador do método, Philippe Emmanuel Souchard descreveu a utilização de posturas específicas para o alongamento global, proporcionando o posicionamento correto das articulações e o fortalecimento dos músculos, visando o restabelecimento do equilíbrio na tensão miofascial das cadeias musculares (SOUCHARD, 1996).

Estudos têm investigado o efeito dessa técnica sobre diversas alterações, como por exemplo, no tratamento da disfunção temporomandibular (MONTEIRO et al., 2013;
MALUF et al., 2010), no tratamento da dor lombar crônica (CASTAGNOLI et al., 2015; LAWAND et al., 2015; LAWAND et al., 2013), no tratamento da dor e da função em pacientes com hérnia de disco lombar (DI CIACCIO et al., 2012), no tratamento da dor, rigidez matinal e mobilidade da coluna vertebral e tórax em pacientes com espondilite anquilosante (SILVA; ANDRADE; VILAR; 2012), no alinhamento espino-pélvico em paciente com espondilolistese (BARROQUEIRO; MORAIS, 2014) e no tratamento da discinesia escapular associada à dor cervical (AMORIM et al., 2015).

Recente revisão sistemática (FERREIRA et al., 2016) que abordou os efeitos da RPG sobre o sistema músculo-esquelético, apresentou resultados referentes a dor lombar, espondilite anquilosante, dor cervical, desordem temporomandibular e síndrome da dor patelofemoral. No entanto, nessa revisão, não foram revisados os tratamentos das alte-

DOI: https://doi.org/10.25110/arqsaude.v21i2.2017.6043

${ }^{1}$ Graduada em Fisioterapia pelo Instituto Porto Alegre.

${ }^{2}$ Graduada em Fisioterapia pela Universidade Católica de Pelotas e doutoranda em Ciência do Movimento Humano pela UFRGS. Autor correspondente: Endereço: Av. Domingos de Almeida, 2187. Bairro Areal - Pelotas/RS. CEP: 96085-470. E-mail: julianasedrez@gmail.com

${ }^{3}$ Graduado em Educação Física pela Universidade do Vale do Rio dos Sinos e doutorando em Ciências da Saúde pela Universidade Federal de Goiás

${ }^{4}$ Doutora em Ciência do Movimento Humano pela UFRGS e docente do curso de fisioterapia e educação física, mestrado e doutorado da UFRGS. 
rações posturais, visto que são escassos os estudos que abordam esse aspecto.

Nesse sentido, sabendo-se que as alterações posturais manifestam-se em grandes proporções na infância e na adolescência (SANTOS et al., 2009; ROSA et al., 2016) e que o RPG visa ao reequilíbrio das cadeias musculares, entende-se que essa técnica poderia trazer benefícios para essa população. Não obstante, poucos estudos abordaram o tratamento da hipercifose em adolescentes (PITA, 2000; COMERLATO; SCANEGATTA; ROSSET, 2013) e ainda permanecem importantes questões a serem respondidas, como os efeitos agudos e a manutenção dos efeitos entre as sessões. Assim, o objetivo desse estudo foi verificar os efeitos agudos e os efeitos de 10 sessões da RPG em um paciente adolescente, do sexo feminino, com hipercifose torácica, a partir de um estudo de caso.

\section{Metodologia}

Este estudo é do tipo estudo de caso (THOMAS; NELSON, 2012). Participou do estudo um único indivíduo, paciente de uma Clínica de Fisioterapia de Porto Alegre. O paciente foi escolhido de acordo com os seguintes critérios: a) estar cursando o Ensino Fundamental; b) possuir de 9 a 15 anos; c) apresentar hipercifose torácica; e d) consentir em participar do estudo por meio do Termo de Consentimento Livre e Esclarecido, assinado pelos pais ou responsáveis.

O estudo foi aprovado pelo Comitê de Ética em Pesquisa da Universidade Federal do Rio Grande do Sul, número 20967 e respeitou o recomendado pela Declaração de Helsink de 1975, revisada em 2000 e a Resolução 466/2012 do Conselho Nacional de Saúde do Brasil.

\section{Tratamento de RPG}

O paciente foi submetido a 10 sessões, com duração de uma hora (20 minutos para as avaliações posturais antes e após sessão e 40 minutos para o tratamento), com intervalo de uma semana entre as sessões, sendo aplicado o método RPG, o qual parte do seguinte princípio: estiramento muscular ativo, realizando o alongamento conjunto dos músculos estáticos antigravitacionais, rotadores internos e os inspiratórios. O tratamento foi aplicado por um fisioterapeuta, com formação e experiência no método.

As 10 sessões foram compostas por duas posturas: (1) postura "rã no chão de braços abertos", sendo o sujeito posicionado em decúbito dorsal, com os braços a aproximadamente $45^{\circ}$ abdução, com as palmas das mãos voltadas para cima; pelve retrovertida, membros inferiores em flexão com abdução e rotação externa de quadril, joelhos fletidos e pés com as regiões plantares em contato uma com a outra (Figura 1a), com progressão para extensão e adução dos membros inferiores e abdução de membros superiores (Figura 1b); e (2) postura "em pé contra a parede", em que o paciente posiciona-se na posição ortostática, dorso em contato com a parede, membros superiores ao longo do corpo com leve abdução, membros inferiores em semiflexão de quadril e joelho em abdução e rotação externa e pés em abdução em $15^{\circ}$ com contato de calcâneos (Figura 1c). Estas posturas foram mantidas durante 4 séries de 2 minutos e 30 segundos, e 3 minutos de intervalo entre as séries, totalizando em média 20 minutos em cada postura. Durante as posturas o fisioterapeuta executou comandos verbais de alinhamento e alongamento axial, respiração e correções de movimentos compensatórios.

Figura 1: (a) Postura "rã no chão de braços abertos" em sua posição inicial; (b) Postura "rã no chão de braços abertos" em sua posição avançada e (c) Postura "em pé contra a parede".



Os pesquisadores não tiveram qualquer influência sobre o método de execução do tratamento de RPG, sendo apenas responsáveis pelas avaliações ao longo do período de tratamento.

\section{Instrumentos e procedimentos de coleta e análise de da- dos}

A avaliação da coluna torácica foi realizada utilizando o instrumento flexicurva (1) antes do início do tratamento (pré-teste); (2) antes e após cada sessão de tratamento; e (3) uma semana após a interrupção do tratamento (pós-teste). Esse intervalo de tempo foi dado com o intuito de evitar que apenas o efeito imediato (agudo) fosse observado nessa última avaliação, visto que para este objetivo, já haviam sido realizadas as avaliações imediatamente após cada sessão. $\mathrm{O}$ pós-teste foi realizado 11 semanas após a avaliação inicial.

As avaliações com o flexicurva foram realizadas sempre pelo mesmo avaliador treinado. Além disso, foi realizada avaliação antropométrica (peso e estatura) e utilizado uma anamnese, baseada no questionário BackPEI (NOLL et al., 2013), para avaliar a presença de dor nas costas e obter informações sobre hábitos comportamentais (prática de atividade física, horas assistindo televisão, utilizando o computador e horas de sono). Exceto a avaliação da coluna torácica, todas as demais avaliações foram realizadas apenas no pré-teste, com o objetivo de caracterizar o participante.

O instrumento de avaliação da coluna torácica, o flexicurva (HINMANN, 2004; TEIXEIRA; CARVALHO, 2007), consiste em uma régua flexível constituída de um metal revestido em plástico. Para a avaliação da coluna torácica o paciente foi posicionado em ortostase, braços relaxados ao longo do corpo, pés paralelos e com o dorso despido. $\mathrm{O}$ avaliador, a partir do método palpatório, demarcou os processos espinhosos das vértebras C7 e T12 do paciente. Logo após, o flexicurva foi moldado sobre a coluna torácica do paciente (Figura 2a) e seu contorno traçado sobre um papel milimetrado (Figura 2b). 
Figura 2: Avaliação com o flexicurva: (a) Molde do flexicurva sobre a coluna torácica e (b) identificação das variáveis: comprimento (XTOTAL), comprimento até o ápice da curvatura (XMEIO) e largura do $\operatorname{arco}(\mathrm{H})$.



A partir do contorno desenhado no papel milimetrado foi mensurado tanto o comprimento do arco vertebral através da ligação das duas extremidades por uma reta, quanto à largura da curvatura (Figura 2b). A partir dessas medidas obteve-se o valor de XTOTAL, referente ao comprimento, de C7 a T12. Ainda, foram mensurados os valores de H que consiste na maior distância da curvatura com a reta que une $\mathrm{C7}$ a T12, e XMEIO, determinada pela distância entre o ponto de T12 e o ponto onde H toca com a reta que une C7 a T12. As variáveis XTOTAL, XMEIO e H foram inseridas em uma equação matemática (Equação 1), que a partir de um polinômio de $3^{\circ}$ grau, fornecia o valor angular da cifose torácica (TEIXEIRA; CARVALHO, 2007)

\section{Equação 1 (TEIXEIRA; CARVALHO, 2007)}

$=180 / \mathrm{PI}()^{*}\left(\mathrm{ATAN}\left(\mathrm{H}^{*} \mathrm{XTOTAL} *(-3 * \mathrm{XMEIO}+2 * \mathrm{XTOTAL}) / \mathrm{XMEIO} /\left(\mathrm{XTOTAL}^{\wedge} 2+\mathrm{XMEIO}^{\wedge} 2-\right.\right.\right.$ $2 *$ XTOTAL*XMEIO) $)$-ATAN $\left(3 * \mathrm{H}^{*}\left(\mathrm{XTOTAL}^{*}{ }^{*} \mathrm{XMEIO}\right) / \mathrm{XMEIO} \wedge 2 /\left(\mathrm{XTOTAL}^{\wedge} 2+\mathrm{XMEIO}^{\wedge} 2\right.\right.$ $2 *$ XTOTAL*XMEIO)*XTOTAL $2-2 * \mathrm{H}^{*}\left(\mathrm{XTOTAL}^{\wedge} 2-3 * \mathrm{XMEIO}^{\wedge} 2\right) / \mathrm{XMEIO}^{\wedge} 2 /\left(\mathrm{XTOTAL}^{\wedge} 2+\right.$ XMEIO^2-2*XTOTAL*XMEIO $) *$ XTOTAL $+\mathrm{H}^{*}$ XTOTAL* $(-3 *$ XMEIO+ $2 *$ XTOTAL $) /$ XMEIO/ (XTOTAL^2+XMEIO^2-2*XTOTAL*XMEIO)))

Para a análise dos dados utilizou-se estatística descritiva, com média, desvio-padrão e frequência. Além disto, para verificar se houve diferenças entre as avaliações da coluna torácica, antes e após cada sessão, foi realizada a média das avaliações antes e após cada sessão, e comparadas a partir do teste U de Mann Whitney. Estas análises foram realizadas no Software SPSS 17.0. O nível de significância foi de 0,05 .

\section{Resultados}

O paciente do sexo feminino, com 14 anos de idade, $43 \mathrm{~kg}$ de massa corporal, 1,65 m de estatura, 15,8 kg/ $\mathrm{m}^{2}$ de IMC. Na anamnese, o participante mencionou que não pratica exercício físico regularmente, assiste em média de 0 a 3 horas de televisão por semana, utiliza o computador por mais de $10 \mathrm{~h}$ por semana, a quantidade de sono foi classificada como intermediária e não sentiu dor nas costas nos últimos 3 meses.

Os resultados referentes aos efeitos agudos das sessões de RPG sobre a cifose torácica demonstraram uma redução média de $5^{\circ}$, o que representa uma redução média de $10,1 \%$ da curvatura (Tabela 1 ). O teste U de Mann Whitney indicou diferença significativa entre pré e pós-teste $(\mathrm{U}=$ $23,5 ; \mathrm{p}=0,045, \mathrm{r}=0,45)$.
Resultado semelhante foi obtido ao se avaliar os efeitos das 10 sessões de RPG sobre a cifose torácica, no qual se obteve $53,9^{\circ}$ e $49^{\circ}$ no pré e pós-teste, respectivamente (Tabela 1), ou seja, uma redução da cifose torácica de $4,9^{\circ}$, representando uma redução de $9,1 \%$ do valor obtido no pré-teste.

Tabela 1: Ângulo da cifose torácica obtido no pré e pós-teste, antes e após cada sessão de RPG e as diferenças angulares e percentuais entre as sessões.

\begin{tabular}{ccccc}
\hline Sessão & \multicolumn{2}{c}{$\begin{array}{c}\text { Angulo da cifose } \\
\text { torácica na sessão }\left({ }^{\mathbf{0}}\right)\end{array}$} & \multicolumn{2}{c}{$\begin{array}{c}\text { Diferenças entre as } \\
\text { sessões }\end{array}$} \\
& Antes & Após & Ângulo & \% \\
\hline Pré-teste & 53,9 & - & & \\
\hline 1 & 53,9 & 34,6 & $-19,3$ & $-35,8$ \\
2 & 49,5 & 43,8 & $-5,7$ & $-11,5$ \\
3 & 45,5 & 35,6 & $-9,9$ & $-21,8$ \\
4 & 49,5 & 46,2 & $-3,3$ & $-6,7$ \\
5 & 49,9 & 52,0 & 2,1 & 4,2 \\
6 & 40,9 & 41,6 & 0,7 & 1,7 \\
7 & 50,3 & 46,6 & $-3,7$ & $-7,4$ \\
8 & 43,4 & 39,7 & $-3,7$ & $-8,5$ \\
9 & 43,7 & 42,8 & $-0,9$ & $-2,1$ \\
10 & 46,2 & 40,3 & $-5,9$ & $-12,8$ \\
\hline Média & $\mathbf{4 7 , 3} \pm \mathbf{4}$ & $\mathbf{4 2 , 3} \pm \mathbf{5}$ & $\mathbf{- 5 , 0}$ & $\mathbf{- 1 0 , 1}$ \\
\hline Pós teste & 49,0 & & & \\
\hline
\end{tabular}

\section{Discussão}

O objetivo deste estudo foi verificar os efeitos do tratamento da RPG em um sujeito com hipercifose torácica, a partir de um estudo de caso. Os resultados do presente estudo indicaram que o valor angular da cifose torácica, que no pré-teste foi de $53,9^{\circ}$, diminuiu para $49^{\circ}$ após o tratamento de 10 sessões de RPG. Estes dados demonstram que o indivíduo antes do início do tratamento tinha uma hipercifose torácica, caracterizada por valor angular maior que $50^{\circ}$ (TEIXEIRA; CARVALHO, 2007). No entanto, obteve-se após o tratamento de RPG uma diminuição considerável na curvatura torácica, visto que diminuiu $4,9^{\circ}$, sendo a cifose torácica do indivíduo classificada como normal.

Considerando o efeito agudo do tratamento de RPG, ou seja, o efeito imediato após cada sessão, verificou-se que houve uma diminuição do ângulo da curvatura torácica em 8 das 10 sessões de RPG, demonstrando uma redução média de $5^{\circ}(10,1 \%$ da curvatura). Apenas nas sessões 5 e 6 nota-se um pequeno aumento do ângulo torácico e, visto que não fora avaliada a dor antes de cada sessão, especula-se que alguns fatores podem ter influenciado estes resultados, tais como: estresse, dor aguda, fadiga, fatores psíquicos, e ainda a própria sessão de tratamento. Apesar disso, estes resultados permitem concluir que, neste caso, uma única sessão de RPG foi eficaz para a diminuição imediata da hipercifose torácica. Entretanto, este efeito agudo, proveniente de apenas de uma sessão, não é suficiente para que o paciente mantenha estes benefícios por uma semana, ou seja, o efeito agudo de uma sessão não é eficaz para a melhora da hipercifose torácica, pois a diminuição da curvatura torácica não se manteve até o 
início da próxima sessão.

Resultados idênticos foram encontrados por Pita (2000), o qual, a partir do tratamento com RPG realizado com um paciente adolescente do sexo masculino com cifose aumentada $\left(55^{\circ}\right)$ e outras alterações posturais (desvio lateral da cabeça, ombros assimétricos, rotação de tronco), adotando três posturas de correção "rã no ar", "rã no chão de braços abertos" "rã no chão de braços fechados", verificou, por meio da radiografia, de testes de flexibilidade e avaliação postural, que houve um alinhamento corporal no plano frontal e sagital, e uma diminuição da cifose torácica em $16^{\circ}$. Corroborando com estes resultados, Itokazu et al. (2011) ao aplicar oito sessões de RPG em duas idosas, utilizando as posturas "rã no chão", "em pé na parede", "rã no ar" e "inclinada para frente" observaram melhora no grau de cifose de ambas as voluntárias (uma com redução de $2^{\circ}$ e outra com redução de $16^{\circ}$ ). Os dados do presente estudo em concordância com a literatura sugerem que a técnica de RPG é eficaz para a diminuição do grau de cifose torácica. No entanto, ainda são necessários estudos com maior número amostral e com maior poder metodológico, como os ensaios clínicos, para dar maior suporte a esta hipótese.

Modificações posturais também foram observadas por Moreira e Soares (2007), em um estudo de caso realizado com cinco pacientes do sexo feminino, na faixa etária de 20 a 30 anos, que foram submetidas ao tratamento de RPG durante 4 meses, utilizando-se as posturas "rã no chão" e "asa delta" ou "bailarina". Seus resultados indicaram que houve melhora na postura ortostática a partir das imagens de radiografia, com relação à neutralidade escapular e a simetria corporal em todos os casos. Barroqueiro e Morais (2014) ao tratar com RPG um jogador de handebol masculino de 15 anos com espondilolistese istêmica de baixo grau, obtiveram redução da lordose lombar, da inclinação sacral e da inclinação pélvica anterior em $17,2^{\circ}, 16,5^{\circ}$ e $15,1^{\circ}$, respectivamente. E relataram que a RPG foi eficaz na alteração do alinhamento espino-pélvico relacionado à espondilolistese istêmica de baixo grau.

Outros desfechos, relacionados ao tratamento da dor, também têm sido abordados com a técnica de RPG. Bonetti et al. (2010) avaliaram pacientes com dor lombar, divididos em dois grupos (um tratado com RPG e outro com exercícios de estabilização lombar), durante cinco semanas e com frequência de duas sessões semanais. Os autores concluíram que o grupo que realizou exercícios de RPG obteve uma melhora significativa na flexibilidade, dor e mobilidade em relação ao grupo que realizou exercícios de estabilidade lombar. Lawand et al. (2015) também avaliaram o efeito da RPG em pacientes com dor lombar crônica comparado a um grupo controle. O grupo intervenção foi tratado com uma sessão semanal, durante 12 semanas e demonstrou melhora na intensidade da dor, capacidade funcional e alguns aspectos da qualidade de vida imediatamente após a intervenção (três meses) e na avaliação após seis meses.

Um aspecto importante que pode ser observado nesse estudo é o fato de que as modificações agudas obtidas em uma sessão de RPG não se mantinham completamente até a próxima sessão (intervalo de uma semana). Nesse sentido, avaliar os efeitos agudos auxilia para um melhor conhecimento da durabilidade dos efeitos da técnica, a fim de que seja possível prescrever um programa de tratamento com maior embasamento teórico. Nesse sentido, sugere-se a realização de estudos que investiguem os efeitos da RPG em programas de tratamento com diferentes intervalos entre as sessões e de durabilidade do tratamento.

Outro aspecto importante é que para tornar possíveis acompanhamentos frequentes dos pacientes na prática clínica, faz-se necessários instrumentos de baixo custo e não invasivos, como é o caso do flexicurva, método de avaliação utilizado no presente estudo, que permite que a avaliação das curvaturas da coluna vertebral seja realizada sucessivas vezes, como por exemplo, antes e após cada sessão, sem risco de uma exposição excessiva de radiação ionizante (HINMANN, 2004; TEIXEIRA; CARVALHO, 2007). Do mesmo modo, em comparação com outros instrumentos de pesquisa, de uso clínico e laboratorial, como a radiografia e a ressonância magnética, o flexicurva é um instrumento de baixo custo, fácil utilização, fácil operacionalização, e ainda, pode ser utilizado por qualquer profissional da área da saúde em suas avaliações, desde que este profissional seja treinado. Além disso, esse instrumento já teve a sua validade concorrente testada e os resultados assinalam uma forte correlação entre os dados obtidos com o flexicurva e com os exames de Raios-X. Isto, juntamente com o excelente nível de reprodutibilidade intra e inter-avaliadores, justifica sua recomendação para uso na prática clínica (de Oliveira et al., 2012).

Salienta-se que o resultado do presente estudo não poder ser generalizado, visto que se trata de um estudo de caso, com um único indivíduo. Do mesmo modo, sugere-se que em estudos futuros, este tipo de pesquisa seja reproduzido em um maior número de sujeitos, e que o tempo de aplicação do tratamento seja maior. Outra sugestão seria a realização de reavaliações após o término da intervenção, com o objetivo de investigar a manutenção dos resultados a médio e longo prazo.

\section{Conclusão}

Os resultados do presente estudo demonstraram que o tratamento de RPG produz um efeito imediato (agudo) de redução da hipercifose torácica no indivíduo avaliado. Do mesmo modo, os resultados também mostraram que o tratamento de RPG de 10 semanas foi eficaz na diminuição da hipercifose torácica, ou seja, redução do ângulo da cifose torácica, passando de uma curvatura aumentada para dentro dos valores de normalidade.

\section{Referências}

AMORIM, C. S. et al. Effectiveness of Global Postural Reeducation compared to Segmental Exercises on function, pain, and quality of life of patients with scapular dyskinesis associated with neck pain: a preliminary clinical trial. J Manipulative Physiol Ther, v. 37, n. 6, p. 441-447, 2014.

BARROQUEIRO, C.; MORAIS, N. V. The effects of a global postural reeducation program on an adolescent handball player with isthmic spondylolisthesis. J Bodyw Mov Ther, v.18, n.2, p.244-258, 2014.

BONETTI, F. et al. Effectiveness of a 'Global Postural Reeducation' program for persistent low-back pain: a non- 
randomized controlled trial. BMC Musculoskelet Disord, v. 11, n. 285 , p. $1-12,2010$.

CASTAGNOLI, C. et al. Effects in short and long term of Global Postural Reeducation (GPR) on chronic low back pain: a controlled study with one-year follow-up. Scientific World Journal, v. 2015.

COMERLATO, T.; SCANEGATTA, S.; ROSSET, D. Efeitos do método de Reeducação Postural Global (RPG) no tratamento da cifose de scheuermann. FisiSenectus, p. 10-19, 2013

DE OLIVEIRA, T. S. et al. Validity and reproducibility of the measurements obtained using the Flexicurve instrument to evaluate the angles of thoracic and lumbar curvatures of the spine in the sagittal plane. Rehabil Res Pract, v. 2012, p. $1-9,2012$.

DI CIACCIO, E. et al. Herniated lumbar disc treated with Global Postural Reeducation. A middle-term evaluation. Eur Rev Med Pharmacol Sci, v. 16, n. 8, p.1072-1077, 2012.

FERREIRA, G. E. et al. Global Postural Reeducation for patients with musculoskeletal conditions: a systematic review of randomized controlled trials. Braz J Phys Ther, v. 20, n. 3, p.194-205, 2016.

HINMANN, M. R. Comparison of thoracic kyphosis and postural stiffness in younger and older women. Spine, v. 4, n. 4, p. 413-417, 2004.

ITOKAZU, C. A. et al. Efeitos da Reeducação Postural Global na hipercifose e nas variáveis respiratórias de idosos - relato de dois casos. Rev Inspirar Mov Saude, v. 3, n. 6 , p. 38-42, 2011.

LAWAND, P. A. et al. Global Postural Reeducation to treat chronic low back pain: randomized, controlled trial. ACR/ ARHP Annual Meeting, 2013.

LAWAND, P. et al. Effect of a muscle stretching program using the global postural reeducation method for patients with chronic low back pain: A randomized controlled trial. Joint Bone Spine, v. 82, n. 4, p. 272-277, 2015.

MALUF, S. A. et al. Global postural reeducation and static stretching exercises in the treatment of myogenic temporomandibular disorders: a randomized study. $\mathbf{J}$ Manipulative Physiol Ther, v. 33, n. 7, p.500-507, 2010.

MONTEIRO, W. et al. Effectiveness of global postural reeducation in the treatment of temporomandibular disorder: case report. J Bodyw Mov Ther, v. 17, n. 1, p.53-58, 2013.

MOREIRA, C. M. C.; SOARES, D. R. L. Análise da efetividade da reeducação postural global na protusão do ombro após a alta terapêutica. Fisioter. mov, v. 20, n. 1, p. 93-99, 2007.
NOLL, M. et al. F. Back Pain and Body Posture Evaluation Instrument (BackPEI): development, content validation and reproducibility. Int J Public Health, v.58, n.4, p.565-572, 2013.

PITA, M. D. C. Cifose torácica tratada com reeducação postural global. Arq. ciências saúde UNIPAR, v. 4, n. 2, p. 159-164, 2000.

ROSA, B. N. et al. Monitoring the prevalence of postural changes in schoolchildren. J Phys Ther Sci, v. 28, n. 2, p. 326-331, 2016.

SANTOS, C. I. et al. Ocorrência de desvios posturais em escolares do ensino público fundamental de Jaguariúna, São Paulo, Brasil. Rev. paul. pediatr, v. 27, n. 1, p. 74-80, 2009.

SILVA, E. M., ANDRADE, S. C., VILAR, M. J. Evaluation of the effects of Global Postural Reeducation in patients with ankylosing spondylitis. Rheumatol Int, v. 32, n. 7, p.2155-2163, 2012.

SOUCHARD, P. E. O stretching global ativo. 2. ed. São Paulo: Manole; 1996

TEIXEIRA, F. A.; CARVALHO, G. A. Confiabilidade e validade das medidas de cifose torácica através do método flexicurva. Rev. bras. fisioter, v. 11, n. 3, p. 199-204, 2007.

THOMAS, J. R.; NELSON, J. K. Métodos de pesquisa em atividade física. 6 ed. Porto Alegre: Artmed, 2012. 315p.

Recebido em: 06/03/2016 Aceito em: 29/06/2016 